\title{
Abramson Cancer Center
}

National Cancer Institute

\section{Source}

National Cancer Institute. Abramson Cancer Center. NCI Thesaurus. Code C39302.

The Abramson Cancer Center at the University of Pennsylvania seeks the eradication of cancer through relentless progress in basic research, innovative translation of new knowledge through clinical trials, and state-of-the-art compassionate cancer care. The goal of the Abramson Cancer Center is to facilitate collaborations that would otherwise not be possible in a traditional cancer center organized by departments. It was designated as a comprehensive cancer center by NCl in 1974 . 